\title{
A Sixteen-channel Phased Array Driving System for Ultrasound Hyperthermia
}

\author{
Bing-Yuh Lu ${ }^{1}$, Win-Li Lin ${ }^{2}$, Yung-Yaw Chen ${ }^{1}$, Te-Son Kuo ${ }^{1,2}$, Cheng-Yi Wang ${ }^{2}$ \\ ${ }^{1}$ Department of Electrical Engineering ${ }^{2}$ Center for Biomedical Engineering \\ National Taiwan University, Taipei, Taiwan, ROC
}

\begin{abstract}
A sixteen-channel driving system for ultrasound hyperthermia has been designed and constructed. This driving system was divided into two modules. Each module contained one main board and eight driving cards. The two modules were connected with a 64-wired flat cable. The main board had nine slots for the driving cards. Each driving card included phase shifter, amplitude controller, power amplifier and power switch. The serial port of a single-chip microprocessor (ATMEL 89C51) on the main board of the master module received the driving system instructions from $\mathrm{PC}$. With these system instructions, the program in PC could select which channels would be turned-on or turned-off. Then, PC could control the power level and relative phase of turned-on channels. The bandwidth, power step, phase step, maximum output power, power stability within 100 minutes and relative phase error for each channel are $0.5-3.0 \mathrm{MHz}, 256,16,18$ Watts, $10 \%$ and $10 \%$, respectively. This system can be extended if needed. Above all, this power driving system can be employed in multi-element transducers for ultrasound hyperthermia and other high power medical therapy.
\end{abstract}

\section{Introduction}

In order to deliver the ultrasound energy into the tumor site and avoid hurting the normal tissue as possible, the ultrasound focusing technique has been applied. There are four methods to focus ultrasound energy: spherical transducer, lens, reflectors and electrical focusing[1]. In these four methods, the electrical focusing is the most flexible to scan by changing the output power and relative phase of the driving signals. The requirements of the driving system for ultrasound phased arrays are as follows: (1) multi-channel power amplifiers to drive multi-element phased arrays, (2) a certain bandwidth to drive different resonant frequency of ultrasound phased arrays, (3) the ability to adjust relative phase and output power of each channel for the scanning ultrasonic focus. Several driving systems for ultrasound hyperthermia have been developed and constructed. Umemura and Cain's sector-vortex array was driven by a 64-channel amplifier system with independent phase and amplitude control of each element. Amplitude and phase resolutions were 3-bit and 4-bit, respectively[2]. Buchanan and Hynynen's 64-channel intracavitary ultrasound phased array was driven by a system provided with $22.5^{\circ}$ phase shift resolution from $0-360^{\circ}$ and 16 steps of duty cycle (amplitude) to vary from $0-100 \%$ of the output power. Due to a lack of commercially available equipment to drive phased arrays[3], we developed a 16-channel driving system with 4-bit phase resolution and 8-bit amplitude resolution for ultrasound hyperthermia.

\section{Materials and Method}

Figure 1 shows the block diagram of this driving system. The power source supplied the DC voltages of $64 \mathrm{~V}, 12 \mathrm{~V},-12 \mathrm{~V}$ and $+5 \mathrm{~V}$. The output of the function generator was a square wave. This square wave was applied as a reference clock in this system. The frequency of the square wave depended on the resonant frequency of the phased array transducers for ultrasound hyperthermia. The personal computer transmitted the system instructions to the driving system. These system instructions could select which channels turned-on or turnedoff. Then, the PC was able to control the power and relative phase of the output signals. The driving system has 2 driving modules. Each module has one main board and 8 driving cards. On the main board, there were one micro-processor, 9 slots for plugged-in driving cards and 3 parallel ports. A parallel port contained power lines, control lines, data bus, address bus and reference clock. By the connection of parallel ports, the driving system could expand module by module.

Each driving card included four parts: phase shifter, amplitude controller, power amplifier and power switch. The phase shifter was a PEEL ( ICT 22CVI0) programmed as a pre-loadable 4-bit counter to achieve $22.5^{\circ}$ phase step. It could be programmed to be a more than 4 bit counter easily. The value of relative phase pre-loaded into the phase controllers of all 16 channels. The output of phase controllers were triggered by the same signal and to change the phase of output simultaneously. The output square wave of phase controller input into the amplitude controller.

Figure 2 shows the simplified circuit of amplitude controller. In this circuit, the voltage of the Vana depends on the digital input of the digital to analog converter AD7524. The MOSFET $\mathrm{K} 214$ is applied as a switch. If the square wave from phase controller was in the situation of 'high', the K214's channel turned on. Then, Vout would almost be grounded if neglected the resistance between $D$ and $S$ in this MOSFET. Else, the K214 cut off. The voltage of Vout was equal to Vana. So the 
Proceedings - 19th International Conference - IEEE/EMBS Oct. 30 - Nov. 2, 1997 Chicago, IL. USA

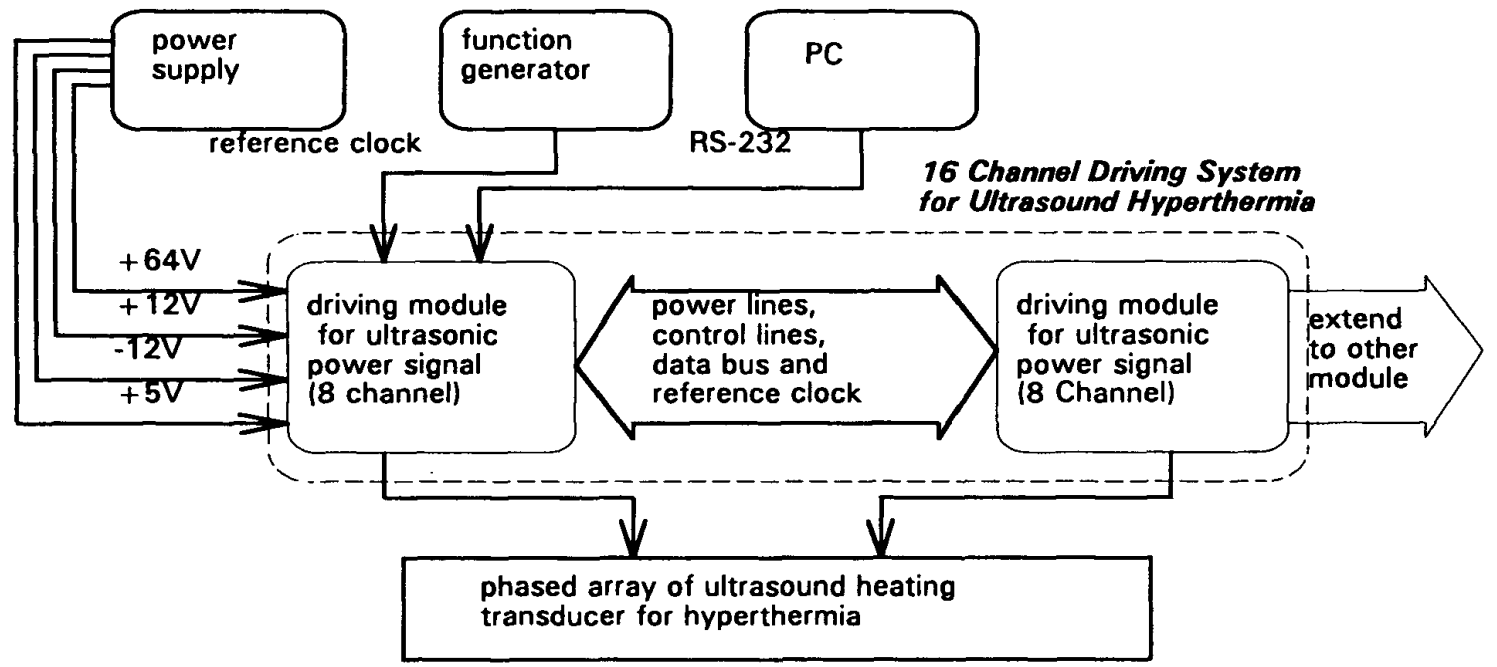

Figure 1 Block diagram of 16 channel driving system for ultrasound hyperthermia

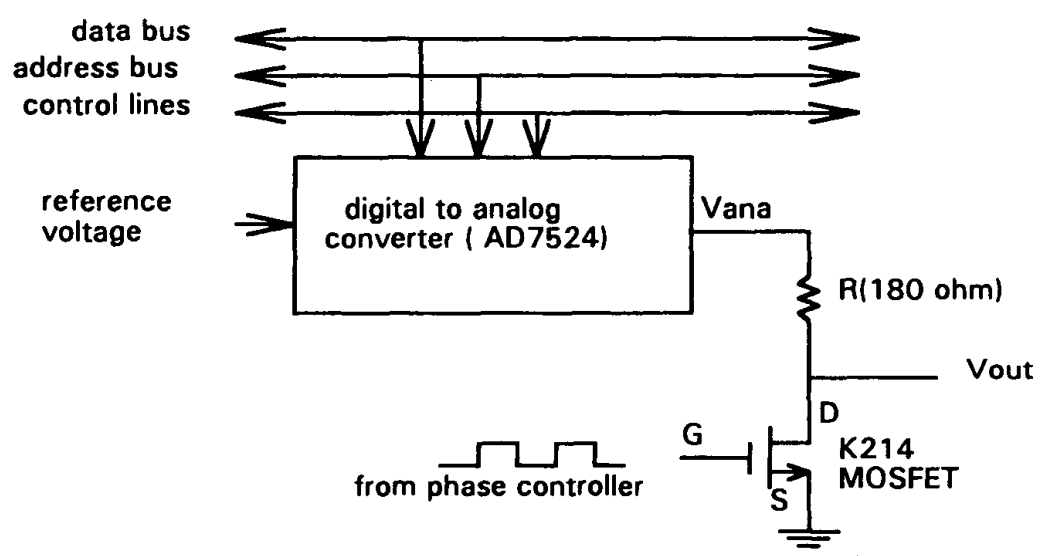

Figure 2 Simplified circuit of the amplitude controller

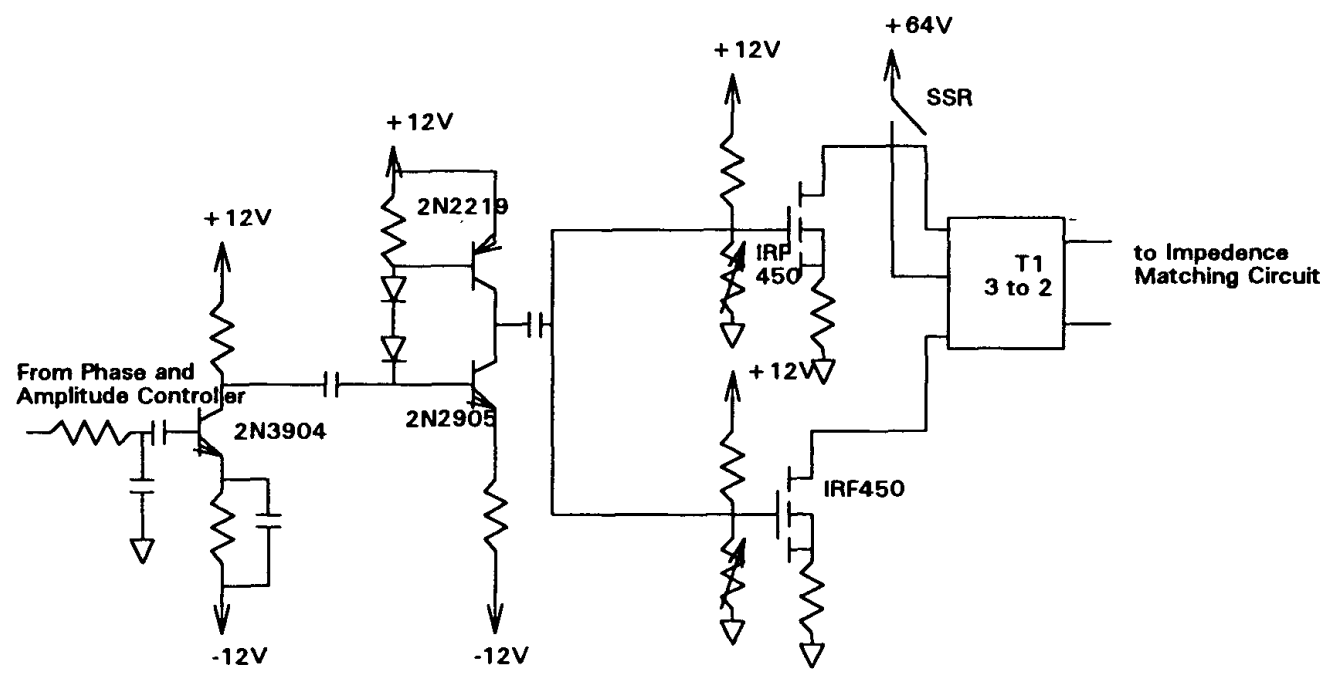

Figure 3 Simplified circuit of the power amplifier 


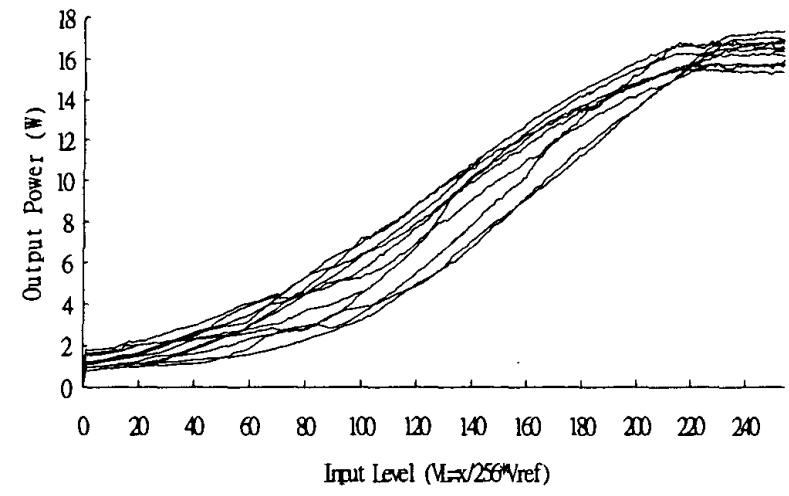

Figure 4 The output power vs. input amplitude level. The voltage of input level $=(x / 256) * 12 \mathrm{~V}$.

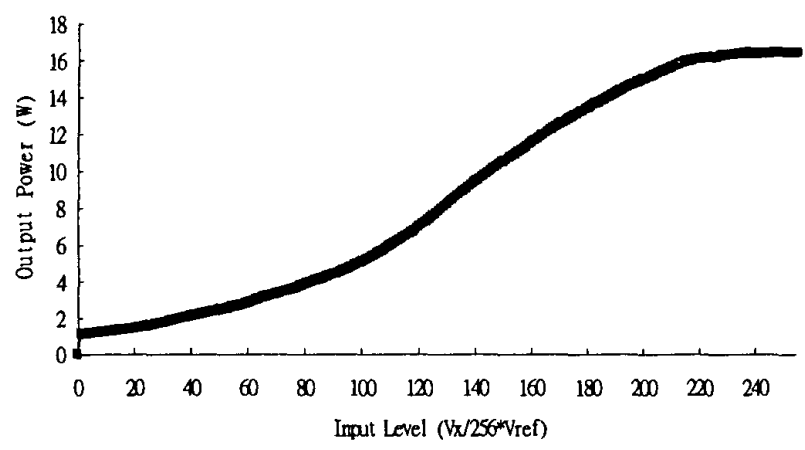

Figure 5 The average of the 16 curves in figure 4 . The maximum variance is $1.5 \mathrm{~W}$.

amplitude of the square wave from phase controller was modulated. The output signal of the amplitude controller (Vout) had been modulated the relative phase and amplitude by phase shifter and amplitude controller. This modulated signal as the input of the power amplifier would decide the relative phase and power of the power amplifier's output.

The simplified circuit of the power amplifier is shown in Figure 3. There are 3 stages in this amplifier: pre-amplifier, push-pull amplifier and class A output stage. The transistor $2 \mathrm{~N} 3904$ as a pre-amplifier operated in common emitter amplifier configuration. In the next stage, transistors $2 \mathrm{~N} 2219$ and 2 N2905 were applied as a push-pull amplifier. The output stage constructed with a pair of RF power MOSFETs (IRF 450) connected in parallel. In this circuit, the solid state relay (SSR) as a power switch of $\mathrm{DC}+64 \mathrm{~V}$ was controlled by the instruction from $\mathrm{PC}$ to decide channels turned-on or turned-off.
The bandwidth, power step, phase step, maximum output power, power stability during 100 minutes and relative phase error for each channel were 0.5-3.0 MHz, 256, 16, 18 Watts, $10 \%$ and $10 \%$, respectively. Figure 4 shows the output power vs. input amplitude of the driving cards from channel 0 to channel 15. Then, we averaged the curves of these 16 channels to obtain the Figure 5. The maximum variance with respect to the curves in Figure 4 is $1.5 \mathrm{~W}$. Figure 6 shows the power deviation during 100 minutes stability test of this system. The maximum variance of the 16 channel is $1 \mathrm{~W}$ (under $10 \%$ ). Figure 7 shows the average relative phase step's errors of all 16 channels. These errors are all under $10 \%$. The frequency response of this system is shown in Figure 8. The bandwidth of this driving system is $0.5-3.0 \mathrm{MHz}$

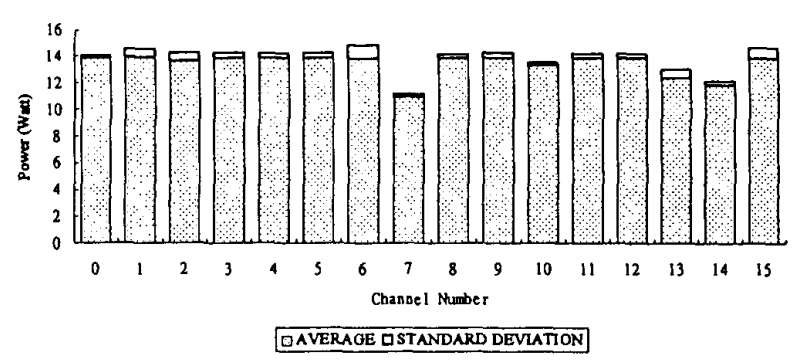

Figure 6 The deviation of the output stability during 100 minutes

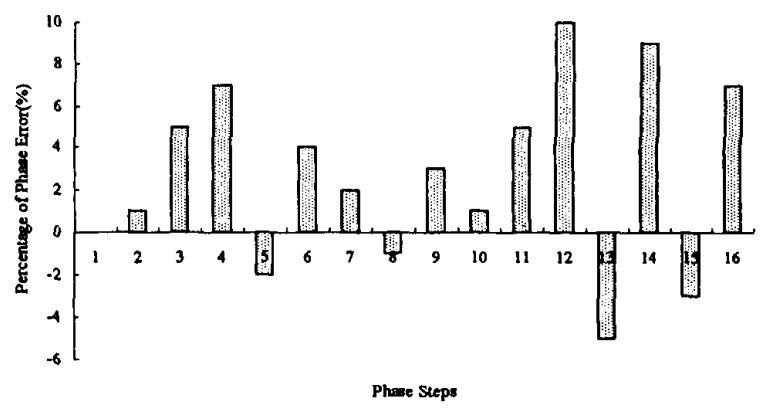

Figure 7 The average of phase step error in the driving system

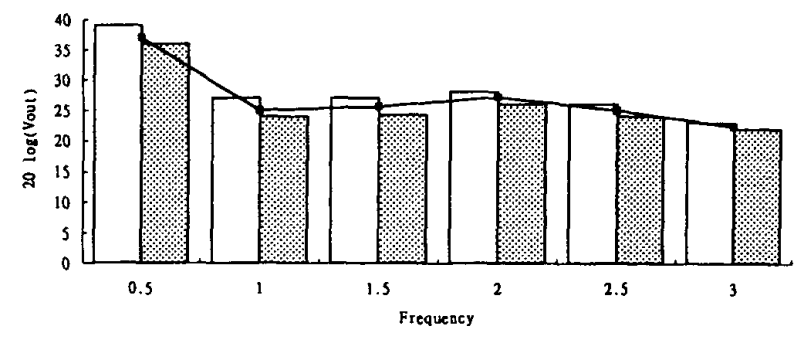

Figure 8 The frequency response of this driving system. 


\section{Discussion}

The output power error between channels could be reduced. If we input the same amplitude level into all of the 16 channels, the output power might not be the same. This is clearly shown in figure 4. To resolve this problem, we can build a look-up table in PC program. Then, we could individually adjust the amplitude level of each channel to approach the same output power.

The relative phase step errors of this driving system could be neglected. In Figure 7, these errors are all under $10 \%$. That is we can precisely change the relative phase of output signal in steps. We have input the same phase level into two channels with $50 \Omega$ resistors, measured the relative phase of output, and found that the difference of phase could be neglected. Thus, the system phase error can be neglected when the system loaded with ultrasonic phased array.

\section{Conclusion}

Considering the system phase error, phase step error, power output error and power stability, this power driving system can be employed in multi-element transducers for ultrasound hyperthermia and other high power medical therapy.

\section{References}

[1] W. L. Lin, R. B. Roemer, E. G. Moros, and K. Hynynen, "Optimization of Temperature Distributions in Scanned, focused Ultrasound Hyperthermia", Int. J. Hyperthermia, vol. 8, pp. 61-78, 1992.

[2] C. A. Cain and S. I. Umemura, "Concentric-Ring and Sector-Vortex Phased-Array Applicators for Ultrasound Hyperthermia", IEEE Trans. Microwave Theo. Tech.vol. MTT-34, pp. 542-551, 1986.

[3] M. T. Buchanan and K. Hynynen, "Design and Experimental of an Intracavitary Ultrasound Phased Array System for Hyperthermia", IEEE Trans. Biomed. Eng. vol. 41, pp. 1178-1187, 1994. 